\title{
Deformidad de la caja torácica
}

\author{
Norman Ramírez-Lluch ${ }^{1}$ y José M. Acevedo-Echevarría ${ }^{2}$
}

\section{Chest wall deformity}

Chest wall deformities are divided as an abnormal development during the growth or those secondary to a congenital malformation. The developmental type is the most common: pectus excavatum or pectus carinatum. The less common are the congenital types of chest wall abnormalities: Poland's syndrome, Jeune's syndrome, espondylothoracic dysplasia, espondylocostal dysplasia and defects of the ribs or sternum. The congenital type usually affects the relationship between the spine, rib cage and the lungs. Therefore, many of these patients will develop a progressive respiratory disturbance of restrictive type known as Thoracic Insufficiency Syndrome. Thoracic insufficiency syndrome is defining as a deficiency of the rib cage to maintain a normal respiration and to sustain the physiological growth of the lungs. In this article will discuss several conditions that will affect the development and function of the chest wall. Key words: spondylocostal dysplasia; spondylothoracic dysplasia; jeune syndrome; poland syndrome.

\section{Resumen}

Las deformidades de la caja torácica se pueden dividir en dos tipos, las que son productos del desarrollo anormal del pecho en el crecimiento y las congénitas que son las secundarias a una malformación estructural del pecho evidente en el nacimiento. Las malformaciones del desarrollo son las más comunes, como por ejemplo pectus excavatum o pectun carinatum. Las menos comunes son las de tipo congénito: síndrome de Poland, displasia espondilotorácica, displasia espondilocostal, síndrome de Jeune y los defectos de la costilla o el esternón. Las deformidades del pecho de tipo congénita se caracterizan por afectar la relación entre la columna vertebral, la caja torácica y los pulmones. La mayoría de estos pacientes desarrollan un disturbio respiratorio progresivo de tipo restrictivo conocido como Síndrome de Insuficiencia Torácica. Este síndrome se define como la deficiencia de la caja torácica para mantener una respiración normal y sostener el crecimiento fisiológico del pulmón. En este artículo discutiremos varias condiciones que afectan el desarrollo y función de la caja torácica.

Palabras clave: displasia espondilotorácica; displasia espondilocostal; síndrome de Jeune; síndrome de Poland.

\section{Introducción}

La posibilidad de evaluar un niño con deformidad de la caja torácica ha ido aumentando con los avances del conocimiento sobre la relación de los pulmones, la caja torácica y la columna vertebral. Las deformidades de la caja torácica no se deben ver solo como un problema estético, sino también como un problema de supervivencia. La mayoría de estos pacientes desarrollan un disturbio respiratorio progresivo de tipo restrictivo conocido como Síndrome de Insuficiencia Torácica ${ }^{1}$.

El Síndrome de Insuficiencia Torácica se define como la deficiencia de la caja torácica en mantener una respiración normal y sostener el crecimiento fisiológico del pulmón ${ }^{1}$. Los pacientes con este síndrome presentan un historial clínico y examen físico compatible con problemas respiratorios, a su vez un examen radiológico que confirmara la deformidad de la caja torácica. Adicionalmente, una tomografía computarizada expondrá un volumen pulmonar deficiente y una función pulmonar limitada. El tratamiento en estos casos consiste en restaurar la función y el volumen torácico, además, de mantener estas correcciones durante el crecimiento $^{2}$. A continuación, discutiremos varias condiciones que afectan el desarrollo de la caja torácica.

\author{
'Hospital de la Concepción, \\ San Germán. \\ 2Ponce Health Sciences \\ University. Ponce \\ Puerto Rico. \\ Recibido el 14 de octubre \\ de 2017 y aceptado para \\ publicación el 6 de diciembre \\ de 2017.
}

Correspondencia a: Dr. Norman Ramírez-LI normanpipe@aol.com 


\section{Displasia espóndilocostal}

La displasia espóndilocostal constituye un desorden genético raramente descrito en la literatura. En el pasado era conocida erróneamente como el síndrome de Jarcho-Levin ${ }^{3}$ debido a la no diferenciación entre la displasia espóndilocostal y la displasia espóndilotorácica. Estas dos condiciones son anatómica y genéticamente diferentes. Esta condición puede ser tanto autosómica dominante, como recesiva con una incidencia de 1 en 40.000 nacimientos vivos. Es causada por un defecto del gen DLL3 del cromosoma 19 q13, el cual está involucrado en las señales de transición epitelial-mesenquimal durante la formación de la columna vertebral ${ }^{4,5}$.

Clínicamente, los pacientes con esta condición tendrán poca estatura de tipo proporcionada, defectos cardíacos, urológicos, hernias inguinales o diafragmáticas y problemas renales. Las radiografías se caracterizan por la presencia de defectos congénitos vertebrales de segmentación o formación tales como: hemivértebras, bloque, barras sin segmentar y, además, defectos intrínsecos de las costillas. Estos defectos intrínsecos de las costillas son: ensanchamiento, bifurcación y/o fusión. En el caso de la displasia espondilocostal los defectos de las costillas serán en un solo hemitórax, a diferencia de la displasia espondilotorácica donde los defectos son en ambos lados del pecho (Figura 1. A-B). La fusión de las costillas causa un anclaje unilateral que propicia el desarrollo de una escoliosis progresiva precoz. Esta escoliosis de desarrollo temprano causará que el lado afectado del pecho inhiba el crecimiento y función normal del pulmón envuelto, lo que con- lleva a que el paciente desarrolle el Síndrome de Insuficiencia Torácica.

\section{Tratamiento}

Actualmente, el tratamiento consiste en una toracotomía abierta del lado afectado con separación de las costillas, inserción del implante conocido como la Costilla Expansora Prostética de Titanio (VEPTR) y alargamiento quirúrgico cada 6 meses (Figura 1.C). El VEPTR es un dispositivo con dos curvaturas que se ajustan a la forma de la caja torácica en la cual está siendo implantado. El mismo es colocado verticalmente a lo largo de la pared pectoral con dos terminales: proximal y distal. El terminal proximal se sujeta a la costilla saludable superior al defecto, mientras que el terminal distal se sujetará a un punto estable que puede ser una costilla, la columna vertebral o la pelvis. Este procedimiento permite el crecimiento normal del pecho afectado, a la vez que corrige la escoliosis de desarrollo temprano ${ }^{6,7}$. Hasta el momento no se ha propuesto alguna otra alternativa como tratamiento para la insuficiencia torácica en este grupo de pacientes. En 2010, Ramírez y cols., publicaron el único estudio que se ha hecho hasta la fecha actual sobre esta técnica quirúrgica en un grupo homogéneo de pacientes espóndilocostales. Todos los pacientes se trataron con una toracotomía de expansión e implantación del VEPTR con sus alargamientos planeados. El objetivo era optimizar la alineación de la columna, mejorar el volumen pulmonar y mantener el crecimiento del pecho. Este estudio reportó un adecuado control de la deformidad de la columna vertebral, un crecimiento torácico efectivo y un mejoramiento
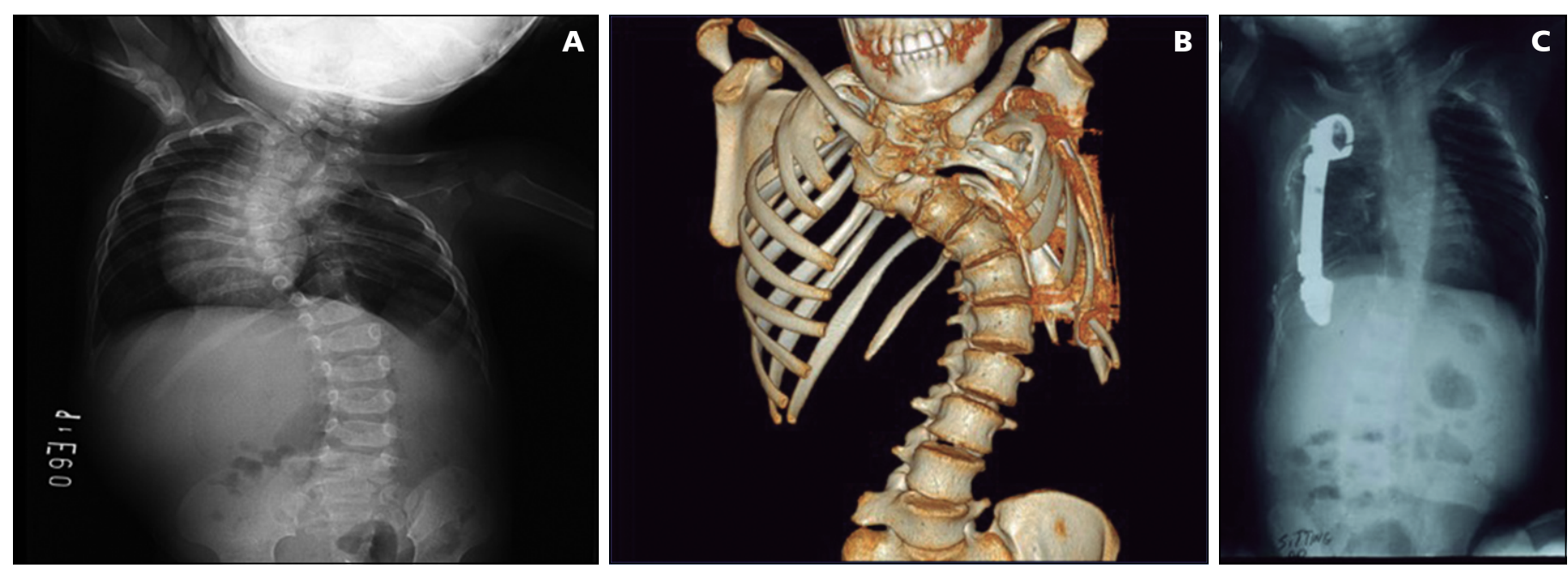

Figura 1. Imágenes de displasia espóndilocostal: A) Radiografía preoperatoria; B) Tomografía computarizada preoperatorio; C) Radiografía posoperativa con VEPTR 
del estatus respiratorio del paciente después del procedimiento con el VEPTR ${ }^{7}$. A pesar de los buenos resultados que se han obtenido con este tratamiento, el VEPTR tiene una serie de desventajas tales como: alta probabilidad de desarrollar infecciones, problemas en la piel y fracturas de costillas inherente a las múltiples cirugías de expansiones. Con el fin de mejorar estas deficiencias, recientemente se desarrolló el MAGEC (Expansión de Control Magnético), dispositivo que contiene un mecanismo de expansión magnético que hace que las barras se extiendan sin necesidad de cirugías. Esto permite alargamientos más frecuentes en la oficina y minimiza los riesgos que conllevan las cirugías repetitivas para un dispositivo como el VEPTR ${ }^{8}$.

\section{Displasia espondilotorácica}

Existe poca literatura mundial sobre esta condición debido a que los pacientes eran agrupados, erróneamente, con los pacientes de displasia espóndilocostal bajo el epónimo de síndrome de JarchoLevin $^{3}$. Esta condición es más frecuente entre los hispanos, siendo el $49 \%$ de los casos reportados de descendencia puertorriqueña ${ }^{9}$. Su transmisión es autosómica recesiva secundaria a un defecto en el gen MEPS2 del cromosoma número $15 \mathrm{q} 26.1^{10}$. Clínicamente, estos pacientes son de poca estatura, cuello corto y rígido, abdomen prominente, extremidades largas para su estatura y hernias inguinales. La radiografía de la columna vertebral se caracteriza por la presencia de múltiples defectos congénitos de las vértebras de formación y/o segmentación tales como: hemivértebras, bloque y barras no segmentadas acompañada de fusión de todas las costillas en ambos lados del pecho (Figura 2A-B). Esta fusión es a nivel de la articulación costo-vertebral, donde las costillas se irradian hacia la parte anterior superior e inferior del pecho. Esta configuración del pecho es conocida en la literatura científica como pecho de abanico o de cangrejo ${ }^{11}$. La fusión de las costillas causa un crecimiento asimétrico entre la columna vertebral y la porción anterior de la caja torácica. Al no crecer la columna vertebral, la caja torácica posterior asume una postura lordótica con una protuberancia anterior del pecho y del abdomen. El pronóstico es pobre, el $45 \%$ de los pacientes fallecen en los primeros 6 meses de edad por fallo respiratorio, pulmonía y/o fallo cardíaco. Esto sucede debido a que las deformaciones de las costillas y vértebras no permiten el crecimiento normal del pecho que a su vez impide el crecimiento y función normal del pulmón (síndrome de la insuficiencia torácica).

\section{Tratamiento}

Actualmente el tratamiento para la displasia espondilotorácica genera controversia. Algunos autores como Mayer, Campbell y Karlin recomiendan cirugía. Esta cirugía consiste en una osteotomía en forma de "V" en la fusión de las costillas para alargar cada hemitórax y la implantación de las VEPTR costilla a costilla ${ }^{8,12,13}$ (Figura 2.C). Por otro lado,

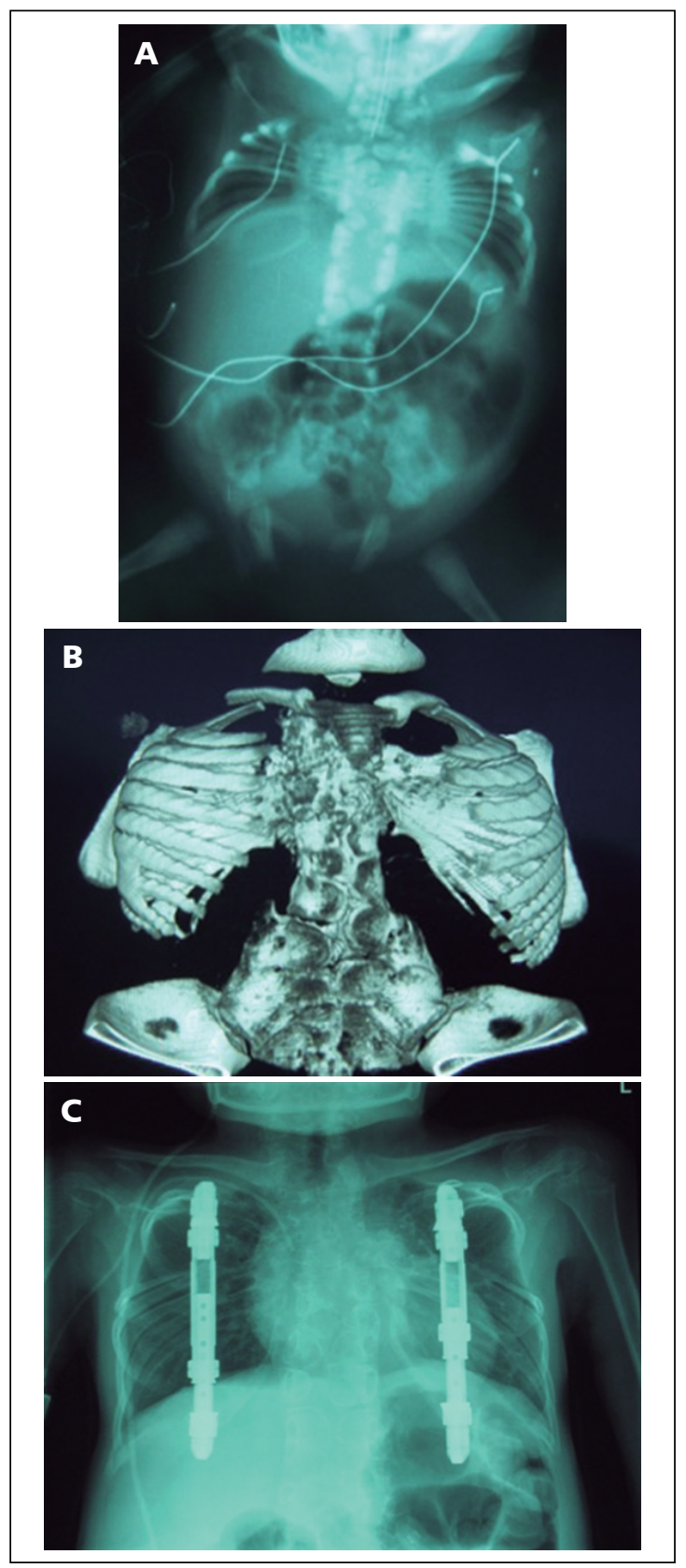

Figura 2. Imágenes de displasia espondilotorácica: A) Radiografía preoperatoria; B) Tomografía computarizada preoperativo; C) Radiografía posoperativa con VEPTR. 
autores como Ramírez y Cornier, no recomiendan intervención quirúrgica alguna. Según estos autores, estos pacientes pueden lograr una buena calidad de vida con un cuidado respiratorio adecuado sin la necesidad de cirugia ${ }^{14,15}$.

\section{Síndrome de Jeune}

Se conoce también como distrofia asfixiante torácica. Es una condición de enanismo sumamente letal con una incidencia de 1 por cada 130.000 nacimientos vivos. Es una condición autosómica recesiva en el cual el gen afectado es el IFT80 responsable por la producción de una proteína de transporte intraflagelar. Este gen está localizado en los cromosomas $15 \mathrm{q} 13$ y el $3 \mathrm{q} 24$.

La presentación primaría del síndrome de Jeune es un hallazgo radiológico en el cual se observa un pecho severamente hipoplástico que puede estar orientado en un patrón de un cilindro angosto o de forma de campana con una hipoplasia más prominente en la parte superior que en la inferior (Figura 3.A-C) ${ }^{4}$. Este síndrome se caracteriza por displasia esqueletales, pecho angosto, micromelia, desórdenes respiratorios y renales. Además, en el $20 \%$ de los casos se ha asociado con polidactilia posaxial de las manos y/o pies 9 . Los disturbios de la caja torácica incluyen hipoplasia pulmonar secundaria a las costillas pequeñas que no crecen, un tórax largo y angosto con una capacidad funcional reducida que no permiten el crecimiento y función normal de los pulmones (síndrome de insuficiencia torácica). A nivel renal presentan enfermedad microcística bilateral que progresa a atrofia tubular y luego a fallo renal. La mayoría de estos pacientes (60\%-80\%) fallecen de fallo respiratorio a temprana edad y los pocos que sobreviven desarrollan fallo renal $^{9}$. Los pacientes con el síndrome de Jeune presentan también problemas de alimentación, prolapso rectal, diarrea, fallo cardiaco, fallo hepático, hinchazón de las extremidades, degeneración de las retinas, malabsorción intestinal y fallo del páncreas ${ }^{16}$.

\section{Tratamiento}

Recientemente, se ha reportado una opción quirúrgica para aumentar el espacio disponible para los pulmones, que le permite un aumento de la expectativa de vida en los pacientes con este síndrome. El procedimiento consiste en una expansión lateral de las costillas dividiendo las costillas de su anclaje anterior y posterior y manteniendo esta expansión con el VEPTR. Este procedimiento se lleva a cabo en el hemitórax derecho debido a que tiene mayor volumen pulmonar. Tres meses más tarde se expande el hemitórax izquierdo de la misma manera. Ambos VEPTR se tienen que expandir quirúrgicamente dos veces al año ${ }^{12,17}$. O'Brein y cols reportaron un aumento de sobrevida de los pacientes de un $70 \%$ y una menor dependencia de ventilador con este tratamiento en comparación con los pacientes sin tratamiento ${ }^{17}$.
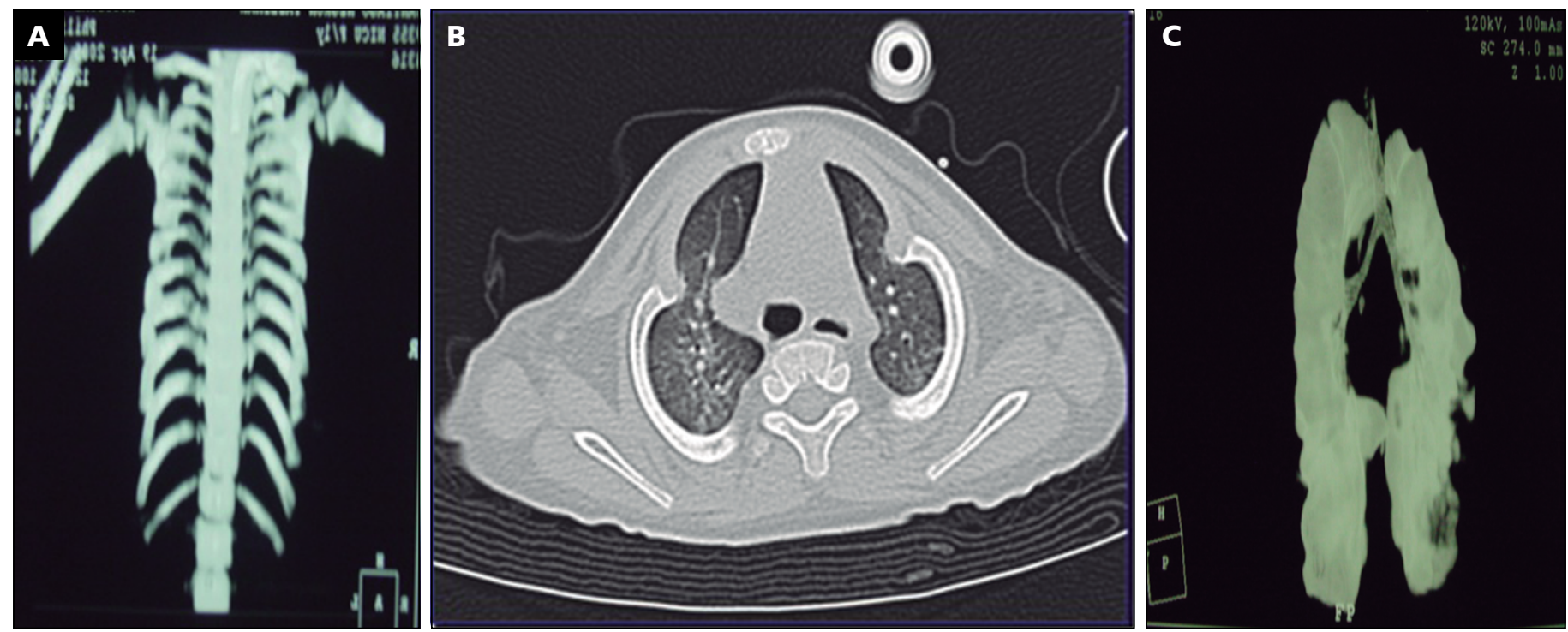

Figura 3. Imágenes de síndrome de Juene: A-B) Tomografía computarizada de la cavidad torácica; C) Tomografía de los pulmones. 


\section{Defectos de esternón}

Los defectos del esternón se pueden clasificar en cuatro tipos. El primero es la ectopia cardiaca torácica en la que hay una ausencia parcial o total de las estructuras sobre el corazón, el cual queda expuesto y tiene un pronóstico pobre. El segundo, ectopia cardiaca cervical, el corazón está desplazado hacia la parte superior, los pacientes sufren de muchas malformaciones craneofaciales y es letal a su vez. El tercero es ectopia cardiaca toracoabdominal donde el corazón está desplazado hacia la parte inferior, cubierto por una fina capa de piel. Tiene mejor pronóstico que los demás defectos. El cuarto es la fisura de esternón o esternón bífido. Esta fisura puede ser completa o parcial. El grado del defecto determinará el pronóstico y el tratamiento ${ }^{18}$. El tratamiento de este cuarto defecto es quirúrgico y diferentes técnicas han sido reportadas en la literatura tales como cierre primario, interposición de tejido óseo o blando, o el uso de próstesis para cubrir el defecto ${ }^{19-22}$.

\section{Anomalías costales}

Las malformaciones aisladas de las costillas son frecuentes, asintomáticas y se encuentran como hallazgos incidentales. Entre las malformaciones más frecuentes están los defectos de número, de forma o ausencia de costillas. Las lesiones óseas son raras y frecuentemente neoplásicas. El 58\% de las lesiones son malignas tales como el sarcoma de Erwings, osteosarcoma y /o condrosarcoma. Las restantes son benignas, siendo las más comunes los osteocondromas o exostosis. Se han reportado neumotórax espontáneos secundarios a los osteocondromas de las costillas (Figura 4.A-B) $)^{23-24}$. El tratamiento está basado en los síntomas y los osteocondromas pueden ser removidos por medio de una toracotomía abierta o toracoscopia asistida por vídeo.

\section{Pectum excavatum}

Es la anormalidad congénita más común de la caja torácica, con una incidencia estimada de 1 en 400 nacimientos vivos y es 5 veces más prevalente en varones que en hembras ${ }^{25}$. Pectum excavatum se caracteriza por la depresión de la pared esternocostal. El cuadro clínico se presenta con el tercio inferior del esternón y los cartílagos centrales hundidos. Muchas veces se nota al nacer, pero si es progresivo se hará más evidente en la pubertad ${ }^{26,27}$. Aún no se conoce de algún defecto genético que cause esta condición. No obstante, se ha observado un patrón de herencia, debido a que aproximadamente un tercio de los pacientes con pectum excavatum tienen familiares con esta condición ${ }^{25}$. Estos casos están frecuentemente asociados a condiciones con anomalías en el tejido conectivo tales como: Marfan, el síndrome de Ehlers-Danlos y el síndrome de Noonan ${ }^{28}$. El 21\% de los casos presenta escoliosis mientras que el $11 \%$ de los pacientes tendrán historial familiar de escoliosis. La literatura más reciente sugiere que muchos de estos pacientes experimentan
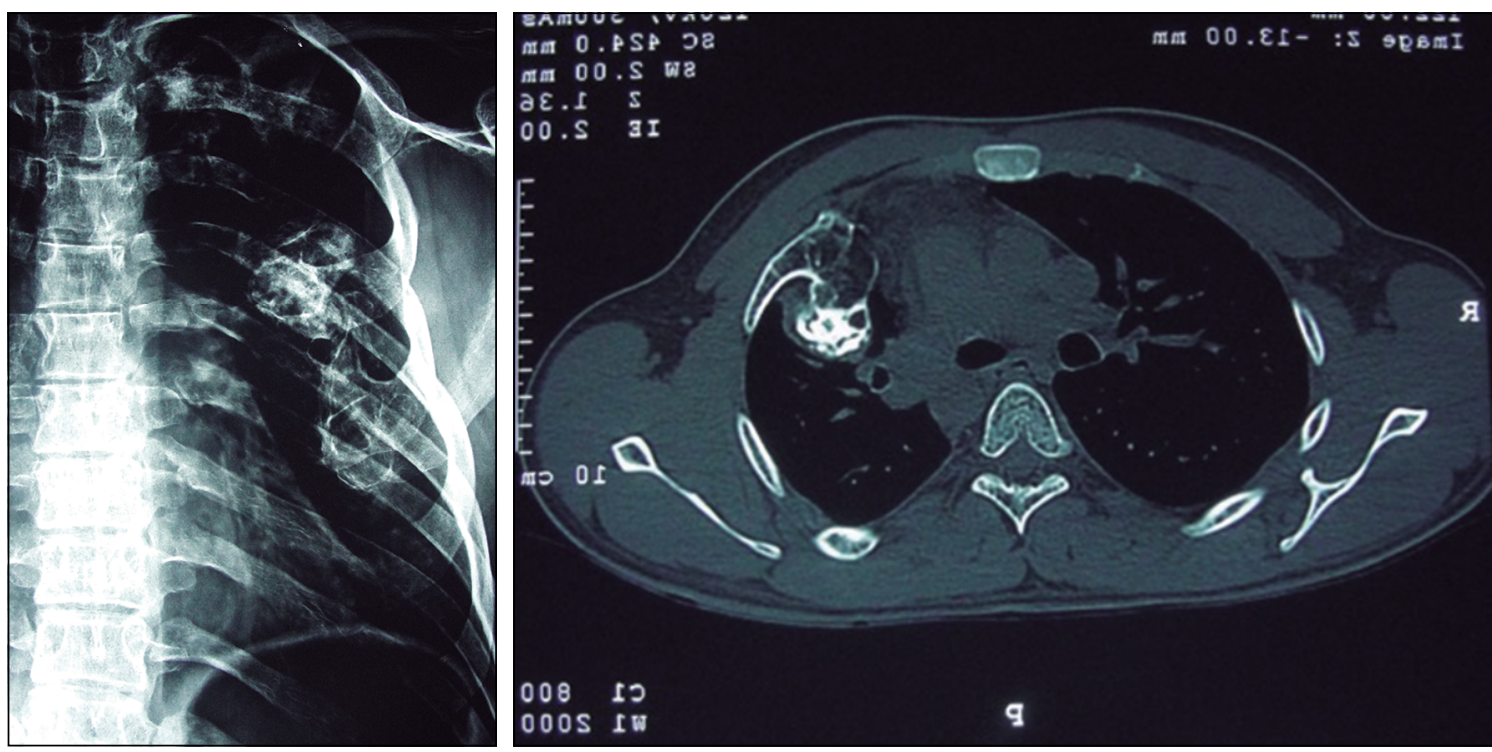

Figura 4. $A / B$ : Imágenes del osteocrondroma costal. A) Radiografía de pecho; B) Tomografía computarizada de pecho. 
cambios perjudiciales a su fisiología cardiovascular y respiratoria durante su crecimiento lo que puede deberse a una reducción en el movimiento de la pared pectoral ${ }^{28}$. Además, dependiendo de la severidad de la deformación y cuan afectadas estén las funciones cardiacas, los pacientes con pectum excavatum pueden desarrollar arritmias cardiacas, disnea, capacidad cardiopulmonar reducida y/o fatiga. También pueden presentar prolapso de la válvula mitral, insuficiencia mitral, y reducción en el llenado y vaciado del lado derecho del corazón que es causado por el efecto de compresión del esternón en ese lado ${ }^{29}$. Algunos tendrán un soplo sistólico secundario a la cercanía del esternón a la arteria pulmonar. A nivel pulmonar estos pacientes también pueden presentar una disminución de su fuerza espiratoria y capacidad vital ${ }^{25}$.

Varios métodos se han descritos para cuantificar la severidad de esta condición. El más utilizado es el método de Haller que consiste en la división de la distancia transversa del pecho sobre la distancia anteroposterior del pecho. Un resultado de 3,25 o más es indicación de tratamiento quirúrgico. Otra indicación quirúrgica es el factor estético de la condición, debido a que estos pacientes están en riesgo de sufrir aislamiento social y a desarrollar tendencias suicidas ${ }^{29,30}$.

\section{Tratamiento}

Por muchos años, los pacientes con pectus excavatum fueron tratados quirúrgicamente usando la técnica abierta de Ravitch introducida en 1949.

Esta técnica consiste en la resección radical de los cartílagos costales, desconexión del esternón de la caja torácica y osteotomía de cuña del esternón ${ }^{31}$. $\mathrm{Su}$ mayor agravante era la creación iatrogénica de una caja torácica rígida y con un limitado potencial de crecimiento. Ante estas complicaciones, Nuss presentó su técnica en 1987 que consiste en introducir una barra detrás del esternón para corregir la deformidad, sin resección de los cartílagos costales u osteotomía del esternón, para así no afectar el crecimiento y flexibilidad de la caja torácica. Después de la descripción inicial, la técnica ha sufrido ciertas modificaciones tales como el incremento de la fortaleza de la barra para mantener la corrección, modificación de los instrumentos para hacerla mínimamente invasiva o en inglés minimally invasive repair of pectus excavatum (MIRPE), se movió de una incisión anterior a la caja torácica a incisiones laterales costales, se añadió el uso de la técnica de toracoscopia y se perfeccionó el método de fijar la barra para así evitar la migración de la barra ${ }^{32}$. La técnica de Nuss, es menos invasiva ya que utiliza una cámara que se introduce por un pequeño orificio para ver la cirugía sin invadir el pecho. Por un hemitórax, se introduce una placa metálica contorneada detrás del esternón, de tal manera que al rotar 180 grados la placa metálica, esta empuja al esternón hacia al frente ${ }^{33}$. Esta placa se remueve después de un mínimo de 2 años, una vez la forma deseada del esternón se ha alcanzado. Los resultados de esta intervención quirúrgica han sido alentadores, ya que los estudios demuestran que hay un mejoramiento en las funciones pulmonares y en la capacidad de ejercicio $^{25}$. Aun así, no podemos olvidar las posibles complicaciones tales como neumotórax, perforaciones cardiacas, daño hepático, gástrico o pulmonar, infecciones, recurrencia, pericarditis y/o distrofia torácica. Entre los factores de riesgos más comunes para tener complicaciones se encuentran haber sido sometido a una cirugía previa del pecho, la severidad de la deformidad, edad del paciente ( $>14$ años) y la falta de experiencia del cirujano ${ }^{34,35}$.

Recientemente, dos estudios de tipo metaanálisis revisaron toda la literatura publicada sobre el procedimiento de Ravitch y sobre el procedimiento de Nuss. El objetivo era evaluar si había diferencia significativa entre ambos procedimientos con relación a la presencia de complicaciones, pérdida de sangre, tiempo quirúrgico y poder así establecer la mejor opción de tratamiento. Interesantemente no encontraron diferencias significativas en relación con la presencia de complicaciones tales como reoperaciones, desplazamiento de la barra, neumotórax, hemotórax, pulmonía o infecciones en la población infantil. En la población adulta los pacientes tratados con el procedimiento de Nuss tuvieron más complicaciones. La técnica de Nuss tuvo dos ventajas: requería menos tiempo quirúrgico y tenía menos pérdida de sangre que el procedimiento de Ravitch ${ }^{36,37}$.

Una alternativa no quirúrgica que se está usando para tratar pectum excavatum, en niños con una deformidad pequeña, es el "Vacuum bell." Es un tratamiento no invasivo que generalmente no va a corregir la deformidad por completo, pero disminuye la profundidad y aplana el pecho. Durante el tratamiento, se aplica una copa de succión para aplicar un vacío y levantar la pared torácica Está indicado en aquellos pacientes que tengan poca deformidad y no desean la cirugía. Está contraindicado en aquellos pacientes con desórdenes musculoesqueléticos, vasculares, coagulopatías o desórdenes cardíacos. Posibles complicaciones con esta técnica conservadora son hematomas subcutáneos, dolor de espalda y/o parestesias transitorias de las extremidades superiores $^{38}$. 


\section{Pectum carinatum}

Es el segundo defecto más común de la caja torácica. Es una protrusión del esternón que se ve al nacer y progresa con el crecimiento ${ }^{39}$. Es una condición de carácter genético. Existen dos tipos de pectum carinatum: condrogladiolar y condromanubrial. La condrogladiolar es aquella donde el gladiolo y los cartílagos inferiores costales sobresalen a través del aspecto más prominente del esternón. Por otro lado, en la condromanubrial la protrusión es en el manubrio y en los cartílagos costales superiores ${ }^{40-42}$. La incidencia de esta condición es aproximadamente de 1 en 1.000 nacimientos vivos ${ }^{28}$, siendo cuatro veces más frecuente en varones que en féminas. Está usualmente asociado a Marfan, síndrome de Morquio, osteogénesis imperfecta, síndrome de Noonan, prolapso de la válvula mitral ${ }^{40} \mathrm{y}$ a escoliosis. Algunos pacientes presentan limitaciones respiratorias, taquipnea y/o excusiones diafragmáticas compensatorias.

\section{Tratamiento}

El tratamiento puede variar desde el uso del sistema dinámico de compresión (DCS) hasta cirugía. El DCS es una ortosis de aluminio que mide y ajusta la presión en la pared torácica, permitiendo una expansión lateral del tórax. Tiende a ser la primera línea de tratamiento, especialmente en pacientes que tienen una pared torácica maleable y sean menores de dieciocho años. Se han reportado una tasa de éxito de $65 \%$ a $80 \%$ con buenos resultados a largo plazo usando este tratamiento ${ }^{43,44}$ (Figura 5).

Por 50 años el tratamiento quirúrgico de predilección fue una modificación del abordaje abierto de tipo Ravitch, que consistía en una resección de la deformidad costal y osteotomía del esternón. Esta técnica tiene múltiples limitaciones tales como extenso tiempo quirúrgico, prolongada estadía hospitalaria, neumotórax, pobre presentación estética y alta tasa de recurrencia. Por lo que, en 2005, Abramson propone su técnica mínimamente invasiva, la cual es una modificación del procedimiento de Nuss. Consiste en introducir una barra metálica en la porción anterior al esternón para comprimir el esternón y corregir la protuberancia costal. La barra metálica se fija a ambos lados de la pared torácica usando placas metálicas ${ }^{42}$. La edad ideal para este procedimiento fluctúa entre los 12 a18 años ${ }^{45}$. La técnica abierta continúa como primera opción en la adultez, si la deformidad es de tipo condromanubrial y es rígida. Si la deformidad es de tipo condrogladiolar y maleable en un joven, entonces la técnica de Abramson puede ser considerada ${ }^{42,45}$.

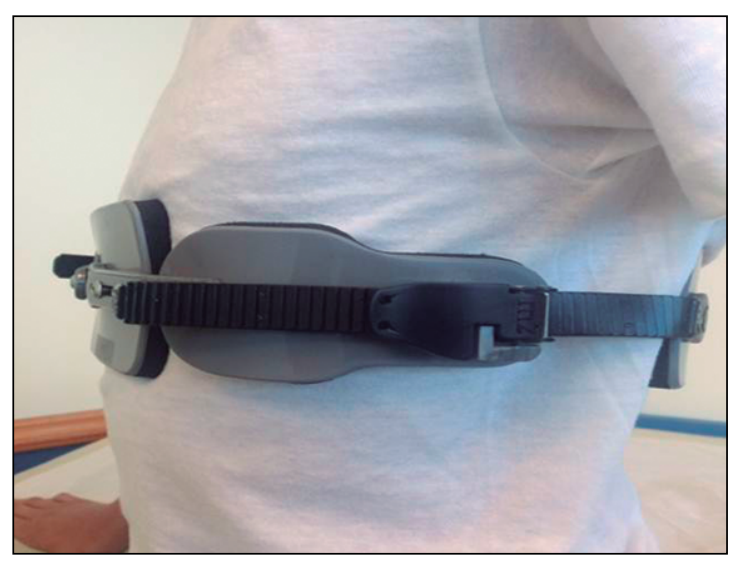

Figura 5. Vista lateral del sistema dinámico de compresión (DCS).

\section{Síndrome de Poland}

Es un espectro de desórdenes que envuelve hipoplasia de la caja torácica. Este desorden puede envolver la ausencia aislada o combinada del pectoral mayor, pectoral menor, serratos anteriores, costillas y/o tejido blando. Tres veces más común en los varones que en mujeres, ocurren en un $75 \%$ en el lado derecho y a veces está asociado a condiciones raras tales como leucemia o síndrome de Mobius ${ }^{46}$. La presentación inicial es variada y estará determinada por el tipo del defecto. La queja principal es cosmética y muy pocos casos tendrán problemas respiratorios. Si hubiese problemas respiratorios serán debido a herniación de los pulmones secundarios a defectos óseos marcados y/o musculares ${ }^{47}$.

\section{Tratamiento}

El tratamiento puede variar desde la observación de los pacientes menos afectados a reconstrucción de la caja torácica. La reconstrucción del defecto de la caja torácica dependerá del defecto anatómico, severidad y género del paciente. En la reconstrucción se puede utilizar: prótesis, colgajos locales o libres, o transferencia del músculo latissimus dorsi, rectus abdominal, glacilis o el uso parcial del latissimus dorsi. Se puede usar también implantes o extensores de tejido blando combinado con el uso de colgajos o grasa local ${ }^{47-50}$.

\section{Responsabilidades éticas}

Protección de personas y animales. Los autores declaran que para esta investigación no se han realizado experimentos en seres humanos ni en animales.

Confidencialidad de los datos. Los autores declaran que en este artículo no aparecen datos de pacientes. 
Derecho a la privacidad y consentimiento informado. Los autores declaran que en este artículo no aparecen datos de pacientes.

\section{Conflicto de intereses}

Los autores declaran no tener conflictos de interés.

\section{Bibliografía}

1. Campbell RM, Smith MD, Mayes TC, Mangos JA, Willey-Courand DB, Kose $\mathrm{N}$, et al. The characteristics of thoracic insufficiency syndrome associated with fused ribs and congenital scoliosis. J Bone Joint Surg Am. 2003;85-A:399-408.

2. Campbell RM, Smith MD. Thoracic insufficiency syndrome and exotic scoliosis. J Bone Joint Surg Am. 2007;89:108-22.

3. Pérez-Comas A, García-Castro JM. Occipito-facial-cervico-thoracicabdomino-digital dysplasia; Jarcho-Levin syndrome of vertebral anomalies. Report of six cases and review of the literature. J Pediatr. 1974;85:388-91.

4. Mayer OH. Chest wall hypoplasiaprinciples and treatment. Paediatr Respir Rev. 2015;16:30-4.

5. Turnpenny PD, Bulman MP, Frayling TM, Abu-Nasra TK, Garrett C, Hattersley AT, et al. A gene for autosomal recessive spondylocostal dysostosis maps to 19q13.1-q13.3. Am J Hum Genet. 1999;65:175-82.

6. Campbell RM, Smith MD, Mayes TC, Mangos JA, Willey-Courand DB, Kose $\mathrm{N}$, et al. The effect of opening wedge thoracostomy on thoracic insufficiency syndrome associated with fused ribs and congenital scoliosis. J Bone Joint Surg Am. 2004;86-A:1659-74.

7. Ramírez N, Flynn JM, Emans JB, Betz R, Smith JT, Price N, et al. Vertical expandable prosthetic titanium rib as treatment of thoracic insufficiency syndrome in spondylocostal dysplasia. J Pediatr Orhop. 2010;30:521-26.

8. Mayer O, Campbell R, Cahill P, Redding G. Thoracic Insufficiency Syndrome. Curr Probl Pediatric Adolesc Health Care 2016;46:72-97.

9. Ramírez N, Villarin S, Ritchie R, Thompson KJ. Thoracic Insufficiency Syndrome: An Overview. Razavi Int $\mathbf{J}$ Med. 2015;3:2-8.

10. Cornier AS, Staehling-Hampton. K, Delventhal KM, Saga Y, Caubet J-F,
Sasaki N, et al. Mutations in the MESP2 Gene Cause Sondylothoracic Dysostosis/ Jarcho-Levin Syndrome. Am J Hum Genet. 2008;82:1334-41.

11. Cornier AS, Ramírez N, Carlo S, Reiss A. Controversies surrounding JarchoLevin syndrome. Curr Opin Pediatr. 2003;15:614-20.

12. Campbell RM, Jr. Spine deformities in rare congenital syndromes: clinical issues. Spine (Phila Pa 1076) 2009;34:1815-27.

13. Karlin JG, Roth MK, Patil V, Cordell D, Trevino H, Simmons J, et al. Management of thoracic insufficiency syndrome in patients with Jarcho-Levin syndrome using VEPTRs (vertical expandable prosthetic titanium ribs). J Bone Joint Surg Am. 2014;96:e181 1-8.

14. Cornier AS, Ramírez N, Arroyo S, Acevedo J, García L, Carlo S, et al. Phenotype characterization and natural history of spondylothoracic dysplasia syndrome: a series of 27 new cases. Am J Med Genet A 2004;128A:120-26.

15. Ramírez N, Cornier AS, Campbell RM Jr, Carlo S, Arroyo S, Romeu J. Natural history of thoracic insufficiency syndrome: a spondylothoracic dysplasia perspective. J Bone Joint Surg Am. 2007;89:2663-75.

16. Tuysuz B, Baris S, AKsoy F, Madazli R, Ungur S, Sever L. Clinical variability of asphyxiating thoracic dystrophy (Jeune) syndrome: Evaluation and classification of 13 patients. Am J Med Genet A 2009;149A:1727-33.

17. O'Brien A, Roth MK, Athreya H, Reinker K, Koeck W, Patil V, et al. Management of thoracic Insufficiency Syndrome in Patients with Jeune Syndrome Using the $70 \mathrm{~mm}$ Radius Vertical Expandable Prosthetic Titanium Rib. J Pediatr Orthop. 2015; 35:783-97.

18. Kotzot D, Schwabegger AH. Etiology of chest wall deformities-a genetic review for the treating physician. J Pediatr Surg. 2009;44:2004-11.

19. S Klein T, Kellner M, Boemers TM, Mack-Detlefsen B. Surgical Repair of a Superior Sternal Cleft in an Infant. European J Pediatr Surg Rep. 2015;3:647.

20. Al-Yamani M, Lavrand F, Thambo JB, Roubertie F. Upper Sternal Cleft With a Complex Congenital Heart Defect: Repair in a Single Stage. Ann Thorac Surg. 2016;101:760-2.

21. Elsayed H, Soliman S. Reversed autogenous sternal plate flaps for treatment of sternal clefts: A novel technique. J Pediatr Surg. 2015;50:1991-4

22. Kaddoura IL, Obeid MY. The cleft sternum: a possible role for pliable prosthetic reconstruction. J Med Liban. 2014;62:232-4.

23. Kim S, Lee S, Arsenault DA, Strijbosh RA, Shamberger RC, Puder M. Pediatric rib lesions: a 13-year experience. J Pediatr Surg. 2008;43:1781-85.

24. Khosla A, Parry RL. Costal osteochondroma causing pneumothorax in an adolescent: a case report and review of the literature. J Pediatr Surg. 2010;45:2250-3.

25. Abdullah F, Harris J. Pectus Excavatum: More Than a Matter of Aesthetics. Pediatr Ann. 2016;45:403-6.

26. Conti M, Cavestri B, Benhamed L, Porte $\mathrm{H}$, Wurtz A. Malformations of the anterior chest wall] Rev Mal Respir. 2007;24:10720.

27. Huddleston CB. Pectus excavatum. Semin Thorac Cardiovasc Surg. 2004;16: 225-32.

28. Mak SM, Bhaludin BN, Naaseri S, Di Chiara F, Jordan S, Padley S. Imaging of congenital chest wall deformities. Br J Radiol. 2016;89:20150595.

29. Obermeyer RJ, Goretsky MJ. Chest wall deformities in pediatric surgery. Surg Clin North Am. 2012;92: 669-84.

30. Kelly RE Jr. Pectus excavatum: historical background, clinical picture, preoperative evaluation and criteria for operation. Semin Pediatr Surg. 2008;17: 181-93.

31. Davis JT, Weinstein S. Repair of the pectus deformity: results of the Ravitch approach in the current era. Ann Thorac Surg. 2004;78:421-6.

32. Nuss D, Obermeyer Rj, Kelly RE. 
Nuss bar procedure past, present and future. Ann Cardiothoracic Surg. 2016;5:422-33.

33. Nuss D. Minimally invasive surgical repair of pectus excavatum. Semin Pediatr Surg. 2008;17:209-17.

34. Pawlak K, Gąsiorowski Ł, Gabryel P, Gałęcki B, Zieliński P, Dyszkiewicz W. Early and late results of the Nuss procedure in surgical treatment of pectus excavatum in different age groups. Ann Thorac Surg. 2016;102:1711-6.

35. Hebra A, Kelly RE, Ferro MM, Yüksel M, Campos JRM, Nuss D. Life-threatening complications and mortality of minimally invasive pectus surgery. J Pediatr Surg. 2018;53:728-32.

36. Kanagaratnam A, Phan S, Tchantchaleishvili V, Phan K. Ravitch versus Nuss procedure for pectus excavatum: systematic review and meta-analysis. Ann Cardiothorac Surg. 2016;5:409-42.

37. Mao YZ, Tang S, Li S. Comparison of the Nuss versus Ravitch procedure for pectus excavatum repair: an updated metaanalysis. J Pediatr Surg. 2017;52:1:54552 .
38. Haecker FM, Sesia S. Vacuum bell therapy. Ann Cardiothorac Surg. 2016;5:440-9.

39. Coelho M, Guimarães P. Pectus carinatum. J Bras Pneumol. 2007;33:463-74.

40. Robicsec F, Watts LT. Pectus carinatum. Thorac Surg Clin. 2010;20:563-74.

41. Park CH, Kim TH, Haam SJ, Jeon I, Lee $\mathrm{S}$. The etiology of pectus carinatum involves overgrowth of costal cartilage and undergrowth of ribs. J Pediatr Surg. 2014;49:1252-8.

42. Katrancioglu O, Akkas Y, Karadayi S, Sahin E, Kaptanoglu M. Is the Abramson technique effective in pectus carinatum repair? Asian J Surg. 2016;1-4.

43. de Beer SA, Gritter M, de Jong JR, van Heurn ELW. The Dynamic Compression Brace for Pectus Carinatum: Intermediate Results in 286 Patients. Ann Thorac Surg. 2017;103:1742-49.

44. Emil S, Sévigny M, Montpetit K, Baird R, Laberge JM, Goyette J, et al. Success and duration of dynamic bracing for pectus carinatum: A four-year prospective study. J Pediatr Surg. 2017;52:124-9.

45. Suh JW, Joo S, Lee GD, Haam SJ, Lee S. Minimally Invasive Repair of Pectus
Carinatum in Patients Unsuited to Bracing Therapy. Korean J Thorac Cardiovasc Surg. 2016;49:92-8.

46. Baas M, Burger EB, Sneiders D, Galjaard RH, Hovius SER, van Nieuwenhoven CA. Controversies in Poland Syndrome: Alternative Diagnoses in Patients With Congenital Pectoral Muscle Deficiency. J Hand Surg Am. 2018;43:186.e1-186.

47. Yiyit N, Işıtmangil T, Öksüz S. Clinical analysis of 113 patients with Poland Síndrome. Ann Thorac Surg. 2015;99:9991004.

48. Romanini MV, Torre M, Santi P. Proposal of the TBN Classification of Thoracic Anomalies and Treatment Algorithm for Poland Syndrome. Plast Reconstr Surg. 2016;138: 50-8.

49. Cherubino M, Maggiulli F, Pellegatta I, Valdatta L. Free gracilis flap for chest wall reconstruction in male patient with Poland syndrome after implant failure. Indian J Plast Surg. 2016;49:249-52.

50. Majdak-Paredes EJ, Shafighi M, Fatah F. Integrated algorithm for reconstruction of complex forms of Poland syndrome: 20year outcomes. J Plast Reconstr Aesthet Surg. 2015;68:1386-94. 